\title{
A Novel Approach for Power Sharing in
}

\section{Microgrids}

\author{
Kartheek Vankadara ${ }^{1}$, M. Purushotham ${ }^{2}$ \\ PG Student, Department of E.E.E. , Shree Institute of Technical Education, Tirupati, India ${ }^{1}$ \\ Assistant Professor, Department of E.E.E. , Shree Institute of Technical Education, Tirupati, India ${ }^{2}$
}

\begin{abstract}
The action of inverters which are connected in parallel in microgrids is supported on droop process. The actual droop process comprises of rectifying the final voltage frequency and size to obtain self-standing power sharing without control wire interconnections. The actual voltage droop process reveals several drawbacks such as interior multiloop feedback control, frequency and voltage deviations. This paper intends a modern control tactic in microgrid applications by bringing down substantial flux in place of inverter final voltage. Firstly, substantial flux, active and reactive powers are mathematically obtained and a relationship is established between them which are employed to promote a modern flux droop technique. A small signal model is improved in direction to sketch the main parameters which are to be controlled and to study the steadiness as well as the system dynamics. A direct flux control step by step technique is used to adjust the substantial flux agreeing to the droop controller to evade the utility of PI controllers and PWM modulators. The simulation output shows that intended flux droop tactic can obtain reactive and active power shared by decreasing the frequency deviated than actual droop process, which spotlights influential usage in applications of microgrids.
\end{abstract}

Keywords: Sharing of active and reactive power; microgrids; flux droop; power quality.

\section{INTRODUCTION}

A MICROGRID is a group of smaller (micro) generators united to territorial low-voltage mesh through converters which comprises of power electronic components. Compared to individual distributed generation (DG) one, microgrids propose several technical benefits in terms of flexibility for controlling and ability to embody renewable energy sources [1]-[3]. But, system stableness and power quality are major issues due to the alternating character of fluctuating load outline and the renewable energy sources. In addition, as the sharpness and the DG units grow, the power converters are directed to act more effectively and powerfully to support dynamic stability and maximum power quality. Modern and professional control techniques are necessary to meet the essentials.

Many inverter control tactics employed in microgrids are to obtain accurate power sharing within the DGs. The superiority of the tactics is traced from non-stoppable power supply control plans. Droop technique is the most familiar tactics in microgrid applications. This universal base is from the theory of power system, where a synchronous generator is joined to grid which makes the frequency to drop when power demand grows. The actual droop process was presented first into microgrids [4], where between inverters; the active power shared is obtained by rectifying frequency and by rectifying the size of inverter final voltage the reactive power shared is obtained. The droop method actually performs with high reliability as it employs only territorial measurements of power.

The actual droop process has many disadvantages like complex interior multiloop feedback control, voltage distortions and frequency. To exhibit mentioned voltage from droop controller, a multiloop feedback control plan is commonly used for controlling the inverters. In the multiloop feedback control, proportional-integral regulators are employed in external voltage loop and interior current loop. Modulation like sinusoidal PWM is needed to produce the ultimate gate drive signals. Due to this effect, this process needs complicated coordinate change, and much tuning attempt is required to insure the stableness of the system, which causes it difficult to fulfill. It is much understood that excellent power sharing can be obtained when employing the actual droop process, which leads to declination of regulation of voltage due to frequency and magnitude of inverter output voltage which are to be governed instantly. The voltage distortion could be disagreeable in certain applications where the main problem is with power quality.

In modern years, more study has to be done to correct voltage droop process so as to gain steadiness of the system. For example, to obtain more transient response, the derivative-integral terms were introduced into droop controller. By decoupling real and reactive powers, a virtual transformation or impedance method were presented for better power sharing. To improve the power quality, an angle controller is introduced by bringing down the inverter final voltage in 
the place of frequency. The disadvantage is that other phase angles of inverter are not assumed. To procure synchronization, GPS signal is being employed [5]. As to counterbalance for voltage distortion caused by characteristics of droop, a multilayer control strategy was presented [6]. The above-mentioned methods are improved with the help of voltage droop method; by using Q-V and P- $\omega$ relationships. So, difficult multiloop feedback loops are unpreventable; useful power sharing can be obtained at cost of deviation due to voltage.

\section{Parallel inverters Line Impedance Common Bus Loads}

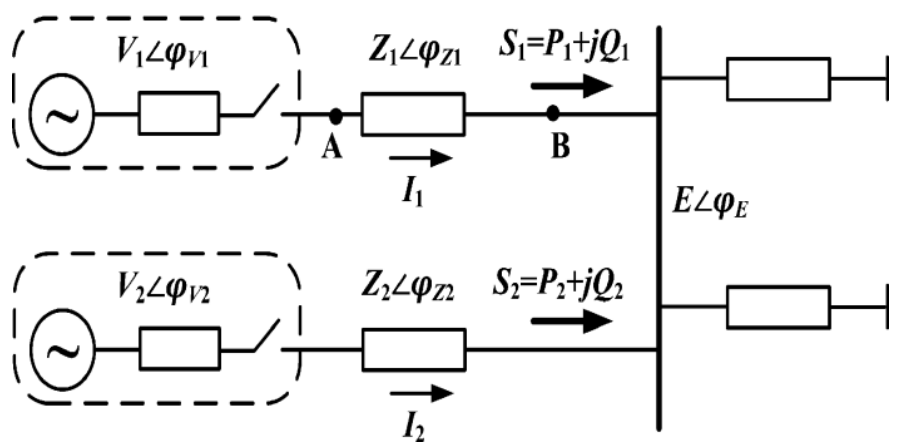

Fig.1 Two parallel connected inverters in microgrids equivalent circuit.

Recently virtual flux droop method is designed which can obtain similar self-governing power sharing to accept droop control of voltage, but frequency deviation very low [7]. The configuration which is to be controlled is simple and no multiloop feedback controls; due to which PI controllers is evaded and PWM modulators are removed. The theory is improved and new tactic is implemented and simulated. The relationship between power flow and inverter flux is deduced which is used for projecting a new virtual flux droop method in next section. The small signal plan can be improved which helps in calculating the control parameters and studies stability of the system in section III. The virtue of intended strategy is projected using MATLAB/SIMULINK in section IV.

\section{PROPOSED DROOP METHOD}

Two distributed generator units which are connected to an AC bus through inverters are shown in figure 1. The system equivalent circuit is described by the mathematical equations which are given by

$$
\begin{aligned}
& V=R I+E+L \frac{d I}{d t} \\
& S=j Q+P=I^{*} E
\end{aligned}
$$

where $\mathrm{I}, \mathrm{V}$ and $\mathrm{E}$ are line current vector, inverter voltage vector and common bus voltage vector on $\mathrm{AC}$ side respectively. The impedance of transmission line is given by $\mathrm{Z}$ where $\mathrm{Z}=(\mathrm{R}+\mathrm{j} \omega \mathrm{L})$. $\mathrm{Q}$ and $\mathrm{P}$ are reactive and real powers flowing from distributed generator to common bus and complex conjugate is denoted by *. The vectors of virtual flux at nodes B and A are given below in the fashion of flux definition as in an electrical machine.

$$
\begin{aligned}
& \psi_{E}=\int_{-\infty}^{t} E d \tau \\
& \psi_{V}=\int_{-\infty}^{t} V d \tau
\end{aligned}
$$

The vectors of converter flux at junction B and of at junction A from (3) and (4) are represented as

$$
\begin{gathered}
\varphi_{f E}=\varphi_{E}-\frac{\pi}{2},\left|\psi_{E}\right|=\frac{|E|}{\omega} \\
\varphi_{f V}=\varphi_{V}-\frac{\pi}{2},\left|\psi_{V}\right|=\frac{|V|}{\omega}
\end{gathered}
$$

where $\varphi_{\mathrm{E}}$ and $\varphi_{\mathrm{V}}$ are phase angles of $\mathrm{E}$ and $\mathrm{V} ; \varphi_{\mathrm{fE}}$ and $\varphi_{\mathrm{fV}}$ are phase angles of $\varphi_{\mathrm{E}}$ and $\varphi_{\mathrm{V}}$. $\omega$ is angular frequency of voltages. Practically R, line resistance is not considered as the impedance of the line is highly inductive. Combining (1), (4) and (3) gives

$$
I=\frac{1}{L} \psi_{V}-\frac{1}{L} \psi_{E}
$$


The apparent power is obtained when substituting (7) into (2) which is given as

$$
S=\frac{1}{L}\left(\psi_{V}-\psi_{E}\right)^{*} E
$$

Also, into (8), substituting (5) and (6) yields

$$
\begin{aligned}
& S=\frac{1}{L}\left(\left|\psi_{V}\right| e^{j\left(\varphi_{V}-\frac{\pi}{2}\right)}-\left|\psi_{E}\right| e^{j\left(\varphi_{E}-\frac{\pi}{2}\right)}\right)^{*} \omega\left|\psi_{E}\right| e^{j \varphi_{E}} \\
& S=\frac{\omega}{L}\left(\left|\psi_{E}\right|\left|\psi_{V}\right| e^{j\left(\frac{\pi}{2}+\varphi_{E}-\varphi_{V}\right)}-\left|\psi_{E}\right|^{2} e^{j\left(\frac{\pi}{2}+\varphi_{E}-\varphi_{E}\right)}\right)
\end{aligned}
$$

So, volt-amps flowing from distributed generation to common AC bus are derived from above equation which yields

$$
S=\frac{\omega}{L}\left|\psi_{E}\right| \psi_{V} \mid \sin \left(\psi_{V}-\psi_{E}\right)+j \frac{\omega}{L}\left(\left.\left|\psi_{E}\right| \psi_{V}\left|\cos \left(\psi_{V}-\psi_{E}\right)-\right| \psi_{E}\right|^{2}\right)
$$

The real and reactive power is obtained with the splitting of above equation into real and imaginary giving

$$
\begin{gathered}
P=\frac{\omega}{L}\left|\psi_{E} \| \psi_{V}\right| \sin \delta \\
Q=\frac{\omega}{L}\left(\left|\psi_{E} \| \psi_{V}\right| \cos \delta-\left|\psi_{E}\right|^{2}\right)
\end{gathered}
$$

where $\delta=\varphi_{\mathrm{V}}-\varphi_{\mathrm{E}}=\varphi_{\mathrm{fV}}-\varphi_{\mathrm{fE}}$. As the angular difference is so small that it is to be assumed as $\sin (\delta)=\delta$ as well as $\cos (\delta)=1$ which yields

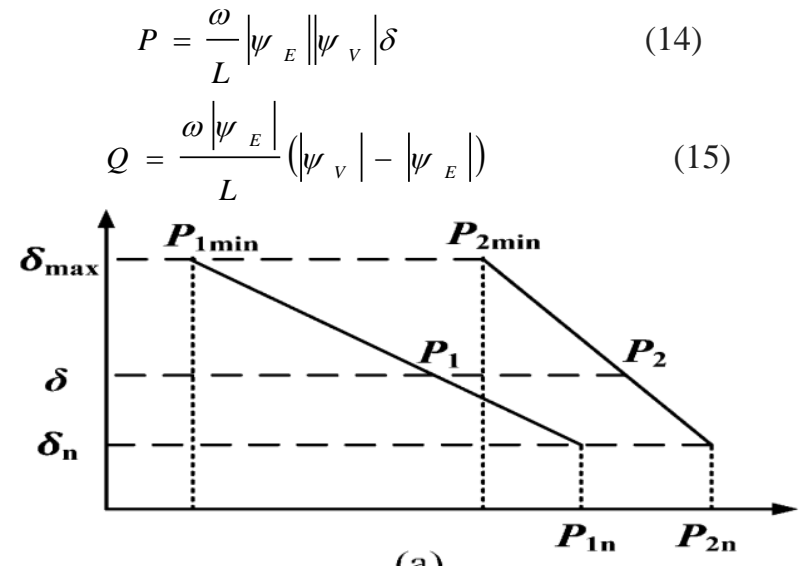

(a)

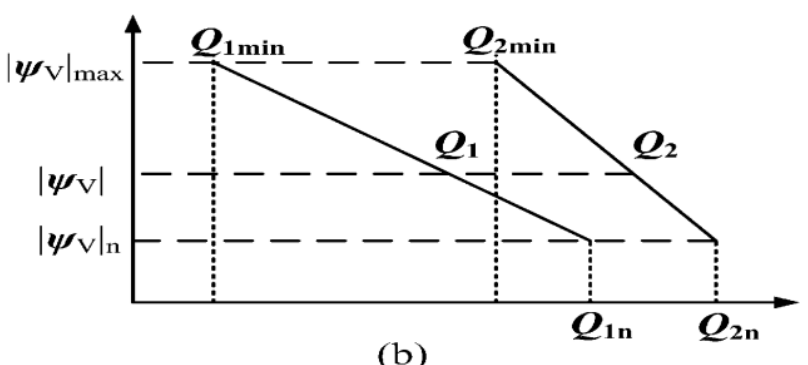

(b)

Fig.2 Real and reactive power shared with the new flux droop method. (a) P- $\delta$ characteristic (b) Q- $\left|\psi_{\mathrm{V}}\right|$ characteristic. So, real power is directly proportional to difference of flux phase angle $\delta$ as well as reactive power is proportional to difference of value of the flux $\left(\left|\psi_{\mathrm{V}}\right|-\left|\psi_{\mathrm{E}}\right|\right)$. Depending on the analysis mentioned, by bringing down the inverter virtual flux a new method is been proposed here which yields

$$
\begin{gathered}
\delta=\delta_{n}-m P_{n}-m P \\
\left|\psi_{V}\right|=\left|\psi_{V}\right|_{n}-n\left(Q_{n}-Q\right)
\end{gathered}
$$


where $\delta_{\mathrm{n}}$ is difference of nominal phase angle between $\psi_{\mathrm{V}}$ and $\psi_{\mathrm{E}}$ and $\left|\psi_{\mathrm{V}}\right|_{\mathrm{n}}$ is inverter flux nominal amplitude. $\mathrm{P}_{\mathrm{n}}$ and $\mathrm{Q}_{\mathrm{n}}$ are power rating of distributed generation units; $\mathrm{n}$ and $\mathrm{m}$ are slopes of characteristics of $\mathrm{Q}-\left|\psi_{\mathrm{v}}\right|$ and P- $\delta$. The proposed technique is shown in figure 2. Real and reactive power are divided between distributed generator units when the load is changed by bringing down $\delta$, difference of their own flux angle and $\left|\psi_{\mathrm{v}}\right|$, value of flux.

\section{SMALL-SIGNAL ANALYSIS}

A small signal is proposed for investigating the system response which allows adjustment of parameters which are to be controlled. The small-signal dynamics of droop controller of P- $\delta$ is obtained when linearizing (12) and (16) which yields

$$
\begin{aligned}
\Delta \delta(s)= & \Delta \delta_{n}(s)-m\left(\Delta P_{n}(s)-\Delta P(s)\right) \\
\Delta P(s) & =G_{p} . \Delta \delta(s) \\
& \text { where } G_{p}=\frac{\omega}{L}\left|\psi_{E}\right|\left|\psi_{V}\right| \cos \delta .
\end{aligned}
$$

The low-pass filters are modeled as first-order approximation which helps in calculation of instantaneous real power where the droop controller of $\mathrm{P}-\delta$ equivalent circuit results from signal model as shown in below figure(a) where $\Delta$ indicates countable values also $\omega_{\mathrm{C}}$ denotes low-pass filters cut-off frequency.

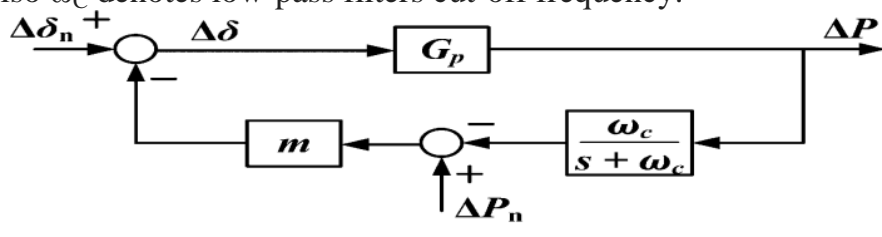

(a)

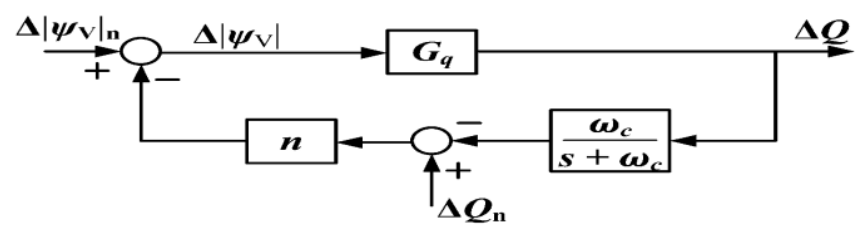

(b)

Fig.3 Small-Signal model (a) Droop controller of P- $\delta$. (b) Droop controller of Q- $\left|\psi_{\mathrm{V}}\right|$.

Using $\Delta \mathrm{P}$ as output and $\Delta \delta_{\mathrm{n}}$ and $\Delta \mathrm{P}_{\mathrm{n}}$ as inputs and by superposition principle, the closed loop transfer function is derived.

$$
\Delta P(s)=\frac{G_{p}\left(s+\omega_{C}\right)}{s+\omega_{C}-\omega_{C} m G_{p}} \Delta \delta_{n}(s)-\frac{m G_{p}\left(s+\omega_{C}\right)}{s+\omega_{C}-\omega_{C} m G_{p}} \Delta P_{n}(s)
$$

Characteristic equation derived from above equation where

$$
s-\omega_{C} m G_{p}+\omega_{C}=0
$$

Eigen value of above expression is expressed like

$$
\lambda_{p}=\omega_{C}\left(m G_{p}-1\right)
$$

The droop controller small-signal dynamics of Q- $\left|\psi_{\mathrm{V}}\right|$ is obtained when linearizing (13), (17) which yields

$$
\begin{aligned}
& \Delta\left|\psi_{V}\right|(s)=\Delta\left|\psi_{V}\right|_{n}(s)-n\left(\Delta Q_{n}(s)-\Delta Q(s)\right) \\
& \Delta Q(s)=\Delta\left|\psi_{V}\right|(s) . G_{q}
\end{aligned}
$$

$$
\text { where } G_{q}=\left|\psi_{E}\right| \frac{\omega}{L} \cos \delta \text {. }
$$

The diiagram of Q- $\left|\psi_{\mathrm{V}}\right|$ droop controller small-signal is shown in above figure(b). Using $\Delta\left|\psi_{\mathrm{V}}\right|_{\mathrm{n}}, \Delta \mathrm{P}_{\mathrm{n}}$ as inputs and $\Delta \mathrm{Q}$ as output by superposition principle, the closed loop transfer function is derived.

$$
\Delta Q(s)=\frac{G_{q}\left(s+\omega_{C}\right)}{s+\omega_{C}-\omega_{C} n G_{q}} \Delta\left|\psi_{V}\right|_{n}(s)-\frac{n G_{q}\left(s+\omega_{C}\right)}{s+\omega_{C}-\omega_{C} n G_{q}} \Delta Q_{n}(s)
$$

The characteristic equation is derived as 
So, the eigen value of (26) yields

$$
\lambda_{q}=\omega_{C}\left(n G_{q}-1\right)
$$

The system eigen value placements vary with the droop slopes $m$ and $n$ from (22) and (27) which show the stability limits for the adjustment of the transient response of the system.

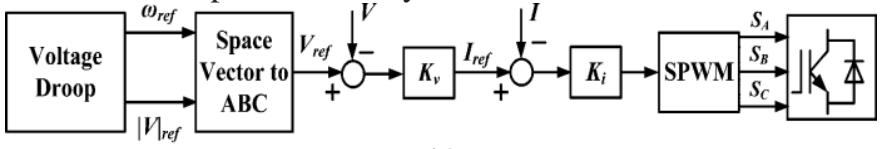

(a)

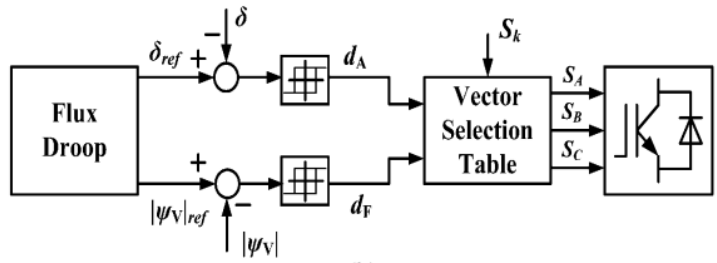

(b)

Fig.4 Inverter control strategies schematic diagram (a) Actual voltage droop method multiloop feedback control. (b) Proposed virtual flux droop method DFC.

\section{CONCLUSION}

The strategy of proposed flux droop control and its performance are simulated with the help of MATLAB/Simulink. Sampling frequency of system is $20 \mathrm{kHz}$ and average switching frequency of every inverter is $3.2 \mathrm{kHz}$.
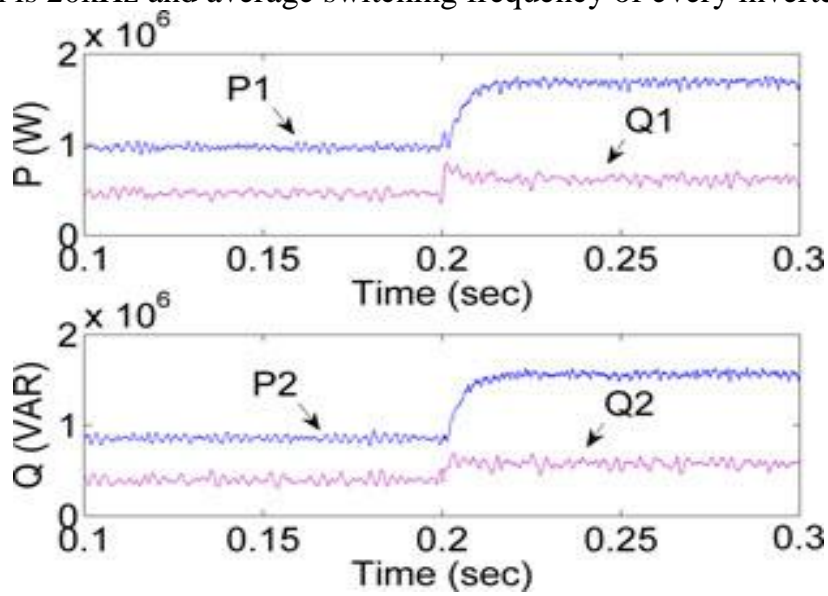

Fig.5 Dynamic response by DGs for real and reactive power being supplied for load changes.

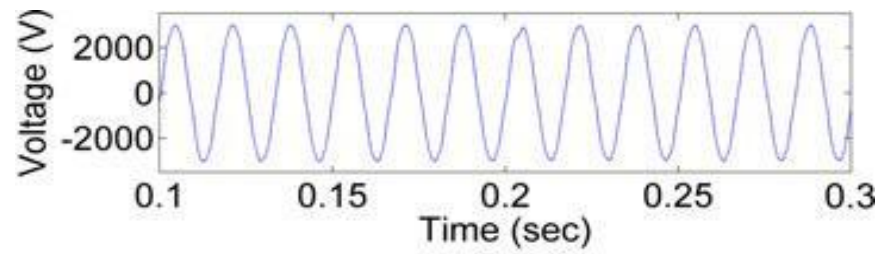

(a)

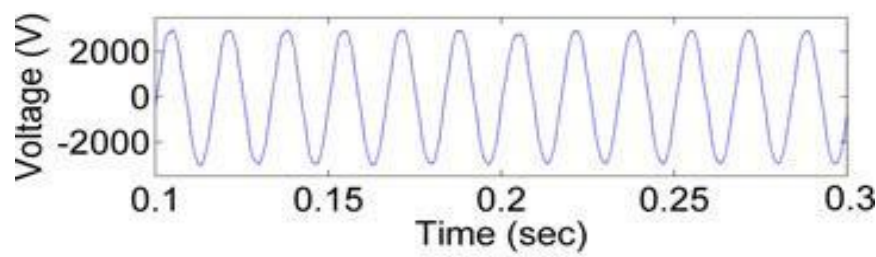

(b)

Fig.6 Load-side voltages performance (a) phase A voltage of $\mathrm{C}_{1}$ (b) phase A voltage of $\mathrm{C}_{2}$. 
The power shared between the two inverters shown in figure 5 is for a step change in load at $0.2 \mathrm{~s}$ which was found that for two DGs to take up load change very fast so that the system reaches steady-point in 10ms. DG1 delivers real power due to steep slope (Section II). The results show the approach towards the novel approach in microgrids applications for sharing power by flux droop method. The load-side performance is shown in figure 6 . The voltage is stable and sinusoidal in nature, before, during as well as after the load changes which is an added advantage for the customers of microgrid.

An approach for controlling power sharing between parallel connected inverters was proposed for microgrid applications in this paper which are different to actual droop method. In proposed droop controller, by bringing down amplitude of the flux and by limiting the phase angle the power sharing is achieved. A DFC algorithm is being introduced in addition which controls inverters to produce required flux from proposed controller. So, feedbacks consisting of PWM modulators and multiloops are not used in control structure. Proposed control strategy is effective and simple which is validated by simulation which highlights the use in microgrid applications.

\section{REFERENCES}

[1]. J. M. Guerrero, F. Blaabjerg, T. Zhelev, K. Hemmes, E. Monmasson, S. Jemei, M. P. Comech, R. Granadino, and J. I. Frau, "Distributed generation: Toward a new energy paradigm," IEEE Mag. Ind. Electron., vol. 4, no. 1, pp. 52-64, Mar. 2010.

[2]. N. Pogaku, M. Prodanovic, and T. C. Green, "Modeling, analysis and testing of autonomous operation of an inverter-based microgrid," IEEE Trans. Power Electron., vol. 22, no. 2, pp. 613-625, Mar. 2007.

[3]. R. Lasseter, "Microgrids," in Proc. IEEE Power Eng. Soc. Winter Meet., 2002, pp. 305-308.

[4]. M. C. Chandorkar, D. M. Divan, and R. Adapa, "Control of parallel connected inverters in standalone ac supply systems," IEEE Trans. Ind. Appl., vol. 29, no. 1, pp. 136-143, Jan./Feb. 1993.

[5]. M. Savaghebi, A. Jalilian, J. C. Vasquez, and J. M. Guerrero, "Secondary control for voltage quality enhancement in microgrids," IEEE Trans. Smart Grid, vol. 3, no. 4, pp. 1893-1902, Dec. 2012.

[6]. Q. Shafiee, J. M. Guerrero, and J. C. Vasquez, "Distributed secondary control for islanded microgrids-A novel approach," IEEE Trans. Power Electron., vol. 29, no. 2, pp. 1018-1031, Feb. 2014.

[7]. J. Hu, J. Zhu, Y. Qu, and J. M. Guerrero, "A new virtual-flux-vector based droop control strategy for parallel connected inverters in microgrids," in Proc. IEEE Energy Convers. Congr. Expo. Asia DownUnder., 2013, pp. 585-590.

[8]. M. P. Kazmierkowski, R. Krishnan, and F.Blaabjerg, Control in Power Electronics. New York: Academic, 2002.

\section{BIOGRAPHIES}

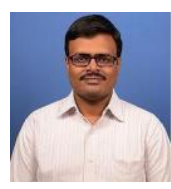

Kartheek Vankadara received B.Tech degree from JNTUH University in 2005 in electrical engineering. Research interests include electric drives, control of power converters, smart grid, microgrid and green energy systems.

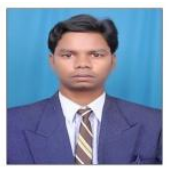

M.Purushotham received M.Tech from JNTUA University during 2011 in power electronics and electrical drives. His research interests include electric drives, control of power converters and artificial intelligence. 